ORIGINAL ARTICLE

\title{
Neonatal resuscitation 3: manometer use in a model of face mask ventilation
}

\author{
C P F O'Donnell, P G Davis, R Lau, P A Dargaville, L W Doyle, C J Morley

See end of article for authors' affiliations

.....................

Correspondence to: Dr O'Donnell, Royal

Women's Hospital

Melbourne, 132 Grattan

Street, Carlton, Victoria

3053, Australia;

colm.odonnell@

rwh.org.au

Accepted 18 March 2005

Published Online First

4 May 2005

Background: Adequate ventilation is the key to successful neonatal resuscitation. Positive pressure ventilation (PPV) is initiated with manual ventilation devices via face masks. These devices may be used with a manometer to measure airway pressures delivered. The expiratory tidal volume measured at the mask $\left(V_{T E(m a s k)}\right)$ is a good estimate of the tidal volume delivered during simulated neonatal resuscitation. Aim: To assess the effect of viewing a manometer on the peak inspiratory pressures used, the volume delivered, and leakage from the face mask during PPV with two manual ventilation devices in a model of neonatal resuscitation.

Methods: Participants gave PPV to a modified resuscitation mannequin using a Laerdal infant resuscitator and a Neopuff infant resuscitator at specified pressures ensuring adequate chest wall excursion. Each participant gave PPV to the mannequin with each device twice, viewing the manometer on one occasion and unable to see the manometer on the other. Data from participants were averaged for each device used with the manometer and without the manometer separately.

Results: A total of 7767 inflations delivered by the 18 participants were recorded and analysed. Peak inspiratory pressures delivered were lower with the Laerdal device. There were no differences in leakage from the face mask or volumes delivered. Whether or not the manometer was visible made no difference to any measured variable.

Conclusions: Viewing a manometer during PPV in this model of neonatal resuscitation does not affect the airway pressure or tidal volumes delivered or the degree of leakage from the face mask.

A bout 3-5\% of newborn infants (4-7 million world wide) are resuscitated at delivery each year. ${ }^{1}$ Internationally agreed consensus statements ${ }^{23}$ and various guidelines advise how to resuscitate newborn infants. ${ }^{4-8}$ All agree that effective ventilation is the key to neonatal resuscitation. All recommend that positive pressure ventilation (PPV) is given with a manual ventilation device through a face mask and that chest wall movement is assessed to monitor the adequacy of ventilation.

Different types of manual ventilation devices (self inflating and flow inflating bags $^{2-8}$ and $T$ pieces ${ }^{58}$ ) have been described. There is a dearth of evidence about which manual ventilation device is superior for the resuscitation of infants at delivery. This is reflected by the considerable variation in the devices used to resuscitate newborns, both within countries and world wide. ${ }^{9}{ }^{10}$ The self inflating bag appears to be the most popular manual ventilation device for resuscitation of the newborn..$^{9-11}$ Despite not being described in international consensus guidelines, use of a modified $\mathrm{T}$ piece is common. ${ }^{10}$

Each type of manual ventilation device may be used with a manometer to measure the airway pressures delivered. Self inflating bags are most often used without a manometer, whereas the use of a manometer is recommended when a flow inflating bag $^{23}$ or T piece device ${ }^{7}$ is used. The effect that a manometer has on the pressures and volumes delivered and leakage from the face mask during PPV at neonatal resuscitation is unclear. We have demonstrated in a model of neonatal resuscitation that the expiratory tidal volume measured at the mask $\left(\mathrm{V}_{\mathrm{TE}(\text { mask })}\right)$ is a good estimate of the tidal volume leaving the lung. ${ }^{12}$ We hypothesised that the use of a manometer would lead to more accurate delivery of target airway pressures. We also hypothesised that observation of the manometer would distract participants from watching chest wall movement and lead to the delivery of smaller volumes because of larger leaks at the face mask.

The aims of this study were to determine the effect that the use of a manometer would have on the peak inspiratory

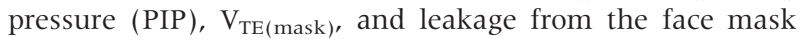
during PPV delivered with manual ventilation devices routinely used at our hospital in a model of neonatal resuscitation.

\section{MATERIALS AND METHODS Setting}

Staff members working at the nurseries of the Royal Women's Hospital, Melbourne, were invited to participate in the study. All had completed the hospital's neonatal resuscitation programme before the study.

\section{Manual ventilation devices and face mask}

We used a Laerdal infant resuscitator (Laerdal Medical, Victoria, Australia) and a Neopuff infant resuscitator (Fisher \& Paykel Healthcare, Auckland, New Zealand). The Laerdal is a silicone $240 \mathrm{ml}$ self inflating bag which automatically reexpands after compression and does not require a gas source to function, although one is normally attached to provide oxygen enriched gas. It does not normally deliver a positive end expiratory pressure (PEEP). ${ }^{13}$ A manometer was placed in the circuit for this study so that the airway pressures generated could be observed. The Neopuff is a modified T piece that requires a compressed gas source, which was set to 8 litres/min for this study. It has a valve on the outlet that is

Abbreviations: PEEP, positive end expiratory pressure; PIP, peak

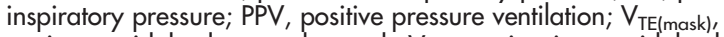

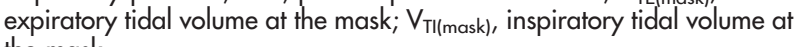
the mask 


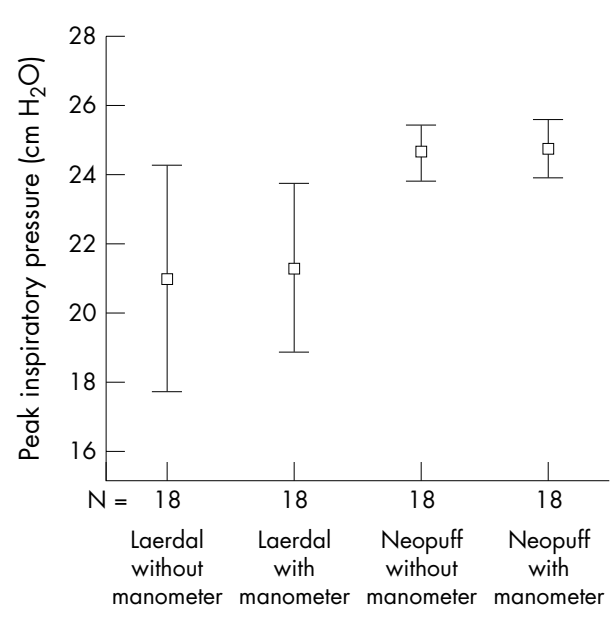

Figure 1 Error bars showing mean and 95\% confidence intervals for mean peak inspiratory pressure delivered with each device used without and with the manometer visible.

adjusted to give a set PEEP for a given rate of flow into the circuit. Occlusion of this valve gives an inflation of a preset PIP. The PIP and PEEP can be observed on the manometer that is built into this device. Both of the Laerdal and Neopuff are routinely used at our hospital, and all participants were accredited to use each of them. We do not routinely use flow inflating bags at our hospital; thus they were not assessed in this study. Each device was used with a size 0/1 round silicone Laerdal face mask (Laerdal Medical), the mask routinely used at our hospital.

\section{Model}

A Laerdal Resusci Baby (Laerdal Medical) resuscitation mannequin was modified by placing a test lung (Dräger, Lubeck, Germany) inside the mannequin's "thorax". The mannequin's "oropharynx" was connected to the test lung so that inflation and deflation caused visible chest excursion similar to that of the unaltered model. Proximal to the test lung, a pressure monitoring line was connected at a $\mathrm{T}$ connector. This system was leak-free and offered a compliance of $0.46 \mathrm{ml} / \mathrm{cm} \mathrm{H}_{2} \mathrm{O}$.

\section{Recording equipment}

We used the Florian Respiratory Monitor (Acutronic Medical Systems, Zug, Switzerland) to measure pressures and gas flow. This monitor measures pressures directly and was calibrated against a column of water. It detects gas flow using a flow sensor with minimal dead space and automatically calculates the volume of gas passing through the sensor by integration of the flow signal. The volume measurement was calibrated against a known volume of gas from a fixed volume syringe. The analogue output from the monitor was connected via an analogue-digital converter to a computer and acquired using the Spectra software program (Grove
Medical, London, UK). The flow, volume, and pressure signals for each inflation were then analysed individually using LabView (National Instruments, Austin, Texas, USA) software and a program specifically developed by one of the investigators (PAD) for the detailed analysis of neonatal respiratory signals.

\section{Values measured}

The flow sensor was placed between each manual ventilation device and the face mask. The monitor thus calculated the volume that passed through the mask towards the mannequin - the inspiratory tidal volume at the mask $\left(\mathrm{V}_{\mathrm{TI} \text { (mask) }}\right)$ and the volume that came back out of the mannequin and through the flow sensor $\left(\mathrm{V}_{\mathrm{TE} \text { (mask) }}\right)$. We have shown that $\mathrm{V}_{\mathrm{TE} \text { (mask) }}$ is a good estimate of the tidal volume at the lung in this model. ${ }^{12}$ Pressures were measured directly using the monitor.

\section{Values calculated}

The face mask leak was the difference between the volume passing through the mask towards the mannequin $\left(\mathrm{V}_{\mathrm{TI} \text { (mask) }}\right)$ and the volume returning from he mannequin through the flow sensor $\left(\mathrm{V}_{\mathrm{TE} \text { (mask) }}\right)$ expressed as a percentage of $\mathrm{V}_{\mathrm{TI}(\text { mask })}$ : leak $(\%)=\left(\left(\mathrm{V}_{\mathrm{TI}(\text { mask })}-\mathrm{V}_{\mathrm{TE}(\text { mask })}\right) / \mathrm{V}_{\mathrm{TI}(\text { mask })}\right) \times 100$.

\section{Instructions}

Participants were requested to give PPV to the mannequin with the Laerdal and the Neopuff, each on two occasionsthat is, four times in all. The manometer could be seen by the participant during one episode of manual ventilation with each device. During the other episode, the manometer remained in each circuit but was covered with opaque cardboard so it could not be seen. The order in which the device was used and whether or not the manometer was visible were allocated randomly. Participants were requested to ventilate the mannequin for two minutes with a PIP of $25 \mathrm{~cm} \mathrm{H}_{2} \mathrm{O}$ and a PEEP of $5 \mathrm{~cm} \mathrm{H}_{2} \mathrm{O}$ and to ensure adequate chest wall movement. Participants were allowed to briefly use each device unblinded to the manometer before starting the study; thus they could preset desired pressures on the Neopuff and estimate the force of squeeze that should be applied to the bag to generate the desired PIP. They could not see the flow, volume, or pressure traces on the Florian monitor or computer during the study.

\section{Statistical analysis}

Data were analysed using SPSS, version 11.5 (SPSS Inc, Chicago, Illinois, USA). Data from participants were averaged for each device used with and without a manometer visible separately. Differences between means were determined using $t$ tests. We tested for equality of variance using the $F$ test. Where there was inequality of variance, we used the $t$ test result that did not assume this equality. Mean differences and 95\% confidence intervals (CIs) were calculated where appropriate. $\mathrm{p}<0.05$ was considered significant.

Table 1 Mean peak inspiratory pressure (PIP), expiratory tidal volume at the mask $\left(V_{T E(m a s k)}\right)$, and leakage from the face mask as a percentage of inflation volume $\left(V_{T I(m a s k)}\right)$ for each device without and with the manometer visible

\begin{tabular}{llll}
\hline & PIP $\left(\mathrm{cm} \mathrm{H}_{2} \mathrm{O}\right)$ & $\mathrm{V}_{\text {TE(mask) }}(\mathrm{ml})$ & Leak $\left(\%\right.$ of $\left.\mathrm{V}_{\text {TI(mask) }}\right)$ \\
\hline Laerdal & $21.0(6.7)$ & $6.3(3.7)$ & $33.0(36.4)$ \\
Laerdal with manometer & $21.3(4.9)$ & $6.2(2.5)$ & $13.6(29.1)$ \\
Neopuff & $24.7(1.6)$ & $5.7(3.9)$ & $34.3(41.1)$ \\
Neopuff with manometer & $24.8(1.7)$ & $5.2(3.5)$ & $35.8(40.8)$ \\
\hline Data are mean (SD). & & &
\end{tabular}




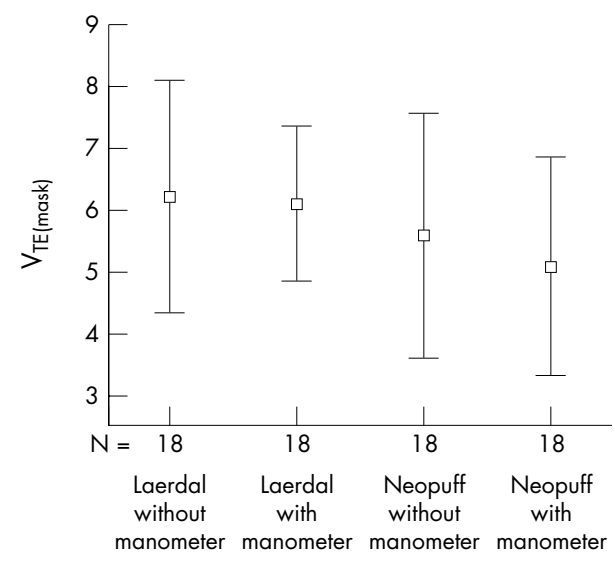

Figure 2 Error bars showing mean and 95\% confidence intervals for mean expiratory tidal volume $\left(\mathrm{V}_{\mathrm{TE}(\text { mask })}\right)$ delivered with each device used without and with the manometer visible.

\section{RESULTS}

\section{Participants}

Eighteen staff members (five consultant neonatologists, three fellows, five residents, and five neonatal nurses) participated in this study.

\section{Measurements}

A total of 7767 inflations were recorded from the 18 participants using each of the four device-manometer/no manometer combinations. This yielded summary statistics for 72 participant-device-manometer/no manometer combinations.

\section{Peak inspiratory pressure (PIP)}

There was significantly greater variability in the PIP delivered with the Laerdal than that delivered with the Neopuff $(F=$ $41.2, \mathrm{p}<0.0001)$, reflected by the wider confidence intervals surrounding the mean PIP delivered with the Laerdal (table 1 , fig 1). The PIP delivered with the Laerdal was lower than that delivered with the Neopuff (mean difference $-3.6 \mathrm{~cm} \mathrm{H}_{2} \mathrm{O}$, $95 \%$ CI -5.6 to $-1.6 ; \mathrm{p}=0.001)$. Whether or not the manometer was visible made little difference in the mean PIP delivered with either device.

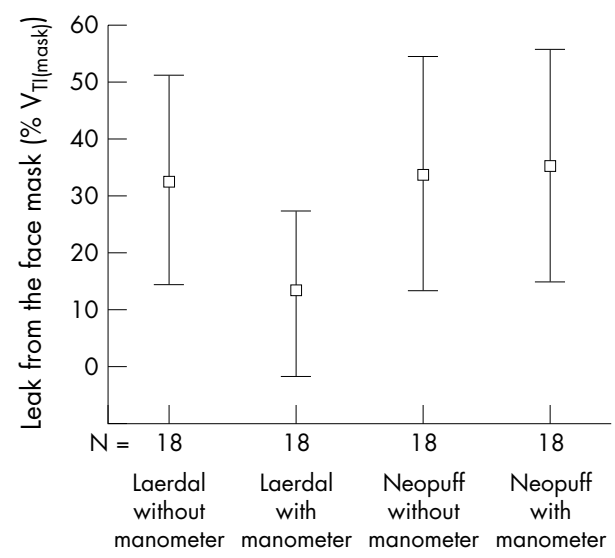

Figure 3 Error bars showing mean and $95 \%$ confidence intervals for mean leakage from the face mask with each device used without and with the manometer visible, expressed as a percentage of inflation volume $\left(\mathrm{V}_{T(\text { mask })}\right)$.
Expiratory tidal volume at the mask ( $\left.\mathrm{V}_{\mathrm{TE} \text { (mask) }}\right)$

There were no significant differences in $\mathrm{V}_{\mathrm{TE} \text { (mask) }}$ delivered between or within devices whether or not a manometer was visible (table 1 , fig 2 ).

\section{Percentage of gas leak from the face masks}

There were no significant differences in the leaks from the face masks between or within devices whether or not a manometer was visible (table 1, fig 3).

\section{DISCUSSION}

We used the most popular resuscitation mannequin, which is used world wide to teach and practise bag-valve-mask ventilation techniques. It is considered to be the most realistic mannequin for the purposes of mask ventilation. ${ }^{14}$ It is unclear, however, how closely this model resembles the newborn infant.

Viewing the manometer did not improve the accuracy with which airway pressures, or tidal volumes, were delivered with either device. Although this is not surprising for the Neopuff, as the desired pressures are preset, it surprised us that the accuracy of delivering a prescribed airway pressure did not improve with the Laerdal bag when the manometer was visible. This finding contrasts with that of a previous study of the impact of a manometer on airway pressures delivered to a mannequin with a self inflating bag. ${ }^{15}$

The rationale for using a manometer during neonatal resuscitation is to avoid giving "excessively high" pressures to the infant, thereby limiting the risk of over-distension of the lung and consequent air leak. There is no evidence that use of a manometer during resuscitation reduces lung damage during mask ventilation. We have shown that the inflating pressure is a poor proxy for the tidal volume delivered during mask ventilation and that the tidal volume delivered is influenced by the face mask leakage. ${ }^{13}$ We have shown that seeing a manometer had no impact on the tidal volumes delivered. Leaks from the face mask were considerable for each device whether or not the manometer was visible. This was consistent with our previous study. ${ }^{13}$ The utility of a manometer during neonatal resuscitation is therefore debatable.

It is commonly believed that, for flow driven devices such as the Neopuff, the manometer allows the operator to detect leaks. However, we have shown that it is possible to deliver

What is already known on this topic

- Manometers are commonly used to monitor the pressures used during PPV with manual ventilation devices and face masks

- Their effect on the pressures used, tidal volumes delivered, and leakage from the mask during neonatal resuscitation is unknown

\section{What this study adds}

- Manometer use did not affect the pressures used, tidal volumes delivered, or the degree of leak from the mask during PPV in this model of neonatal resuscitation

- Leaks are not reliably identified on manometers and the value of a manometer during manual ventilation via a mask is thus unclear 
target airway pressures even when there are substantial leaks at the face mask with this device. ${ }^{13}$ Thus leaks from face masks cannot be reliably detected with manometers. It may be argued that the more important role of the manometer should be to give an estimate of the pressure required to inflate the infants' lungs once the chest is seen to move with manual ventilation, rather than to determine or limit the pressure given. The size and variability of leakage at the mask means that the inflating pressure required to move the chest during mask ventilation is unlikely to be a good estimate of the peak inflating pressure required to ventilate an infant through an endotracheal tube. Furthermore, the clinical assessment of chest wall movement is inferior to direct measurement of the tidal volume delivered in determining appropriate pressures for mechanical ventilation via an endotracheal tube in newborn infants. ${ }^{16}$ Measurement of tidal volume, rather than reliance on observing inflating pressure, during ventilation of infants should be the gold standard irrespective of the setting or device used.

\section{CONCLUSION}

During face mask ventilation of a neonatal resuscitation mannequin, the use of a manometer does not affect the airway pressures used, tidal volumes delivered, or leakage from the face mask. Clinical studies to determine the most effective devices and techniques to give PPV to newborns at delivery are urgently needed.

\section{ACKNOWLEDGEMENTS}

CPFO'D is supported in part by a Royal Women's Hospital Postgraduate Degree Scholarship. PAD and PGD were supported in part by the Murdoch Children's Research Institute. PGD is supported by an Australian National Health and Medical Research Council Practitioner Fellowship.

\section{Authors' affiliations \\ C P F O'Donnell, P G Davis, R Lau, L W Doyle, C J Morley, Royal \\ Women's Hospital Melbourne, Victoria 3053, Australia}

P A Dargaville, Murdoch Children's Research Institute, Melbourne, Victoria 3052, Australia

Competing interests: none declared

\section{REFERENCES}

1 Saugstad OD. Practical aspects of resuscitating asphyxiated newborn infants. Eur J Pediatr 1998;157(suppl 1):S11-15.

2 Kattwinkel J, Niermeyer S, Nadkarni V, et al. ILCOR advisory statement: resuscitation of the newly born infant. Pediatrics 1999;103:e56.

3 International guidelines for neonatal resuscitation: an excerpt from the guidelines 2000 for cardiopulmonary resuscitation and emergency cardiovascular care: international consensus on science. Pediatrics 2000;106:e29.

4 Kattwinkel J, ed. Neonatal resuscitation program: textbook of neonatal resuscitation, 4th ed. Washington DC: American Academy of Pediatrics/ American Heart Association, 2000.

5 Richmond S, ed. Resuscitation at birth: the newborn life support provider manual. London: Newborn Life Support Working Party, Resuscitation Council (UK), 2001

6 Mackway-Jones K, Molyneux E, Phillips B, Wieteska S, eds. Advanced paediatric life support: the practical approach, 3rd ed. London: BMJ Books, 2001.

7 European Resuscitation Council. Recommendations on resuscitation of babies at birth. Resuscitation 1998:37:103-10.

8 Phillips B, Zideman D, Wyllie J, et al. European Resuscitation Council 2000 for newly born life support. A statement from the Paediatric Life Support Working Group and approved by the Executive Committee of the European Resuscitation Council. Resuscitation 2001;48:235-9.

9 O'Donnell CPF, Davis PG, Morley CJ. Neonatal resuscitation: review of ventilation equipment and survey of practice in Australia and New Zealand. $J$ Paediatr Child Health 2004;40:208-12.

10 O'Donnell CPF, Davis PG, Morley CJ. Positive pressure ventilation at neonatal resuscitation: review of equipment and international survey of practice. Acta Paediatr 2004;93:583-8.

11 Palme-Kilander C. Methods of resuscitation in low-Apgar-score newborn infants: a national survey. Acta Paediatr 1992;81:739-44.

12 O'Donnell CPF, Kamlin COF, Davis PG, et al. Neonatal resuscitation 1 evaluating face mask leakage and estimating tidal volume in a model. Arch Dis Child Fetal neonatal Ed 2005;90.

13 O'Donnell CPF, Davis PG, Lau R, et al. Neonatal resuscitation 2: an evaluation of manual ventilation devices and face masks. Arch Dis Child Fetal neonatal Ed 2005;90.

14 Howells R, Madar J. Newborn resuscitation training: which manikin? Resuscitation 2002;54:175-81.

15 Zmora E, Merritt TA. Control of peak inspiratory pressure during manual ventilation: a controlled study. Am J Dis Child 1982;136:46-8.

16 Tracy M, Downe L, Holberton J. How safe is intermittent positive pressure ventilation in preterm babies ventilated from delivery to newborn intensive care unit? Arch Dis Child Fetal Neonatal Ed 2004;89:F84-7. 